\title{
Image Guidance for PICC Insertion: Commentary to "Magnetic Tracking and Electrocardiography-Guided Tip Confirmation System Versus Fluoroscopy for Placement of Peripherally Inserted Central Catheters: A Randomized, Noninferiority Comparison"
}

\author{
Miyuki Sone ${ }^{1}$ (I)
}

Received: 12 July 2020/Accepted: 8 August 2020/Published online: 25 September 2020

(C) Springer Science+Business Media, LLC, part of Springer Nature and the Cardiovascular and Interventional Radiological Society of Europe (CIRSE) 2020

\begin{abstract}
Adequate positioning of catheter tips is crucial for the insertion of central venous catheters, including peripherally inserted central catheters (PICCs), to minimize catheterrelated complications [1-3]. To confirm the location of the tip, imaging is mandatory, and chest radiography is the standard modality. To guide the catheter during the insertion procedure, fluoroscopy is used in the interventional radiology (IR) room due to its real-time visualization capability and prompt confirmation of the catheter. However, bedside insertion without fluoroscopic guidance remains the procedure of choice for critically ill patients as well as physicians or nurses concerned with radiation exposure and staff requirements.
\end{abstract}

Bedside PICC insertion is typically performed using external anatomical measurements or the electrocardiogram (ECG) guidance followed by confirmatory chest radiography $[4,5]$. Recently, a magnetic tip confirmation system (TCS) with real-time ECG guidance was introduced and has been used as an alternative guiding method. Reported accuracy rates for catheter tip insertion ranged from 79.5 to $100 \%$ [3, 4]. In a review of clinical and economic evidence by the National Institute for Health and Care Excellence (NICE), the paucity of the evidence was pointed out [4]. Only a few articles on TCS were published and no comparative study existed.

In the recent issue of CVIR, Mack et al. described the results of a randomized, open-label, single-center, investigator-initiated trial to test the efficacy of TCS in PICC

Miyuki Sone

msone@ncc.go.jp

1 National Cancer Center Hospital, 5-1-1, Tsukiji, Chuo-ku, Tokyo 1040045, Japan insertion compared to fluoroscopy regarding the incidence of correct catheter tip position confirmed by chest radiography [1]. The noninferiority design of this study demonstrated the one-sided $95 \%$ lower confidence limit on the difference between the proportions of two groups as $-23.1 \%(84 / 103,82.4 \%$ in the TCS group; 103/104, $99.0 \%$ in fluoroscopy group). Thus, the noninferiority of TCS to fluoroscopy was not reached. Although this study has inherent limitations (i.e., single-center, performed only by skilled interventional radiologists, lack of cost analysis), the results nullified the hypothesis that, use of TCS is not worse than that of the comparator, fluoroscopy.

The key issue to be addressed regarding this study is how these results will change the clinical practice of PICC insertion. PICCs are used in diverse clinical scenarios. Consequently, PICCs may be inserted in various places including intensive care units, outpatient clinics, bedsides, and radiology departments, and by various operators, such as radiologists, physicians other than radiologists, and nurses [4]. From the evidence of the current study, TCS for PICC insertion is not routinely recommended in the setting where fluoroscopy is available. However, the results do not deny the use of TCS for PICCs, in other settings. The authors noted that TCS would be the most useful in patients where fluoroscopy cannot be used, bedside placement is necessary, or resources are limited.

Interventional radiologists may not be familiar with TCS mainly because we have good access to fluoroscopy for vascular procedures. However, it is always important to be aware of new technologies. Ultimately, these new technologies would better be evaluated compared to the standard techniques using a sound methodology of evaluation by ourselves. Thus, the authors are to be commended for conducting and publishing this randomized controlled 
study. A well-designed RCT can provide credible evidence, and it will strengthen the applied research in our interventional radiology community.

Funding No funding has been received.

\section{Compliance with Ethical Standards}

Conflict of interest The author declares that she has no conflicts of interest.

Ethical Approval This article does not require the approval of the institutional review board.

\section{References}

1. Mack V, Nißler D, Kasikci D, Malouhi A, Aschenbach R, Teichgräber U. Magnetic tracking and electrocardiography-guided tip confirmation system versus fluoroscopy for placement of peripherally inserted central catheters: a randomized, noninferiority comparison. Cardiovasc Intervent Radiol. 2020. https://doi.org/10.1007/s00270-020-02551-0.

2. Tomaszewski KJ, Ferko N, Hollmann SS, et al. Time and resources of peripherally inserted central catheter insertion procedures: a comparison between blind insertion/chest X-ray and a real time tip navigation and confirmation system. Clinicoecon Outcomes Res. 2017;9:115-25.

3. Yamagishi $\mathrm{T}$, Ashida H, Igarashi $\mathrm{T}$, et al. Clinical impact of the Sherlock $3 C^{\circledR}$ tip confirmation system for peripherally inserted central catheters. J Int Med Res. 2018;46(12):5176-82.

4. Dale M, Higgins A, Carolan-Rees G. Sherlock $3 C G((R))$ tip confirmation system for placement of peripherally inserted central catheters: a NICE medical technology guidance. Appl Health Econ Health Policy. 2016;14(1):41-9.

5. Li A, Jiao J, Zhang Y, et al. A randomized controlled study of bedside electrocardiograph-guided tip location technique \& the traditional chest radiography tip location technique for peripherally inserted central venous catheter in cancer patients. Indian J Med Res. 2018;147(5):477-83.

Publisher's Note Springer Nature remains neutral with regard to jurisdictional claims in published maps and institutional affiliations. 